\title{
Core-shell assembly of heteropolyacids and polymer: efficient preparation of cellulose acetate propionate and its processed products
}

\author{
Yunhui $\mathrm{Wen}^{\dagger}$, Huaiying Zhang ${ }^{\dagger}$, Jiao $\mathrm{Li}^{\dagger}$, Sai $\mathrm{An}^{\dagger *}$, Wei Chen ${ }^{\dagger}$ and Yu-Fei Song ${ }^{\dagger *}$
}

†State Key Laboratory of Chemical Resource Engineering, Beijing Advanced Innovation Center for Soft Matter Science and Engineering, Beijing University of Chemical Technology, Beijing 100029, P. R. China.

*E-mail: songyf@mail.buct.edu.cn; Fax/Tel: +86 10-64431832.

Supporting Information: 4 Figures, 4 Tables, 6 pages

\section{List of Contents}

Figure S1 TG-DTA of PS@PMA- $\mathrm{ZrO}_{2}-\mathrm{PW}_{12}$

Figure $\mathbf{S 2}$ TG of CAP film

Figure S3 UV-vis spectrum of CAP film

Figure S4 UV-vis spectra of $\mathrm{PW}_{12}$ and the reaction solution after separation of the used-PS@PMA$\mathrm{ZrO}_{2}-\mathrm{PW}_{12}$

Table S1 Effect of HPAs on esterification activity, $M w$ and viscosity of CAP

Table S2 Effect of temperature on esterification activity, $M w$ and viscosity of CAP

Table S3 Effect of esterification time on esterification activity, $M w$ and viscosity of CAP

Table S4 Effect of anhydride/cellulose mass ratio on esterification activity, $M w$ and viscosity of CAP 


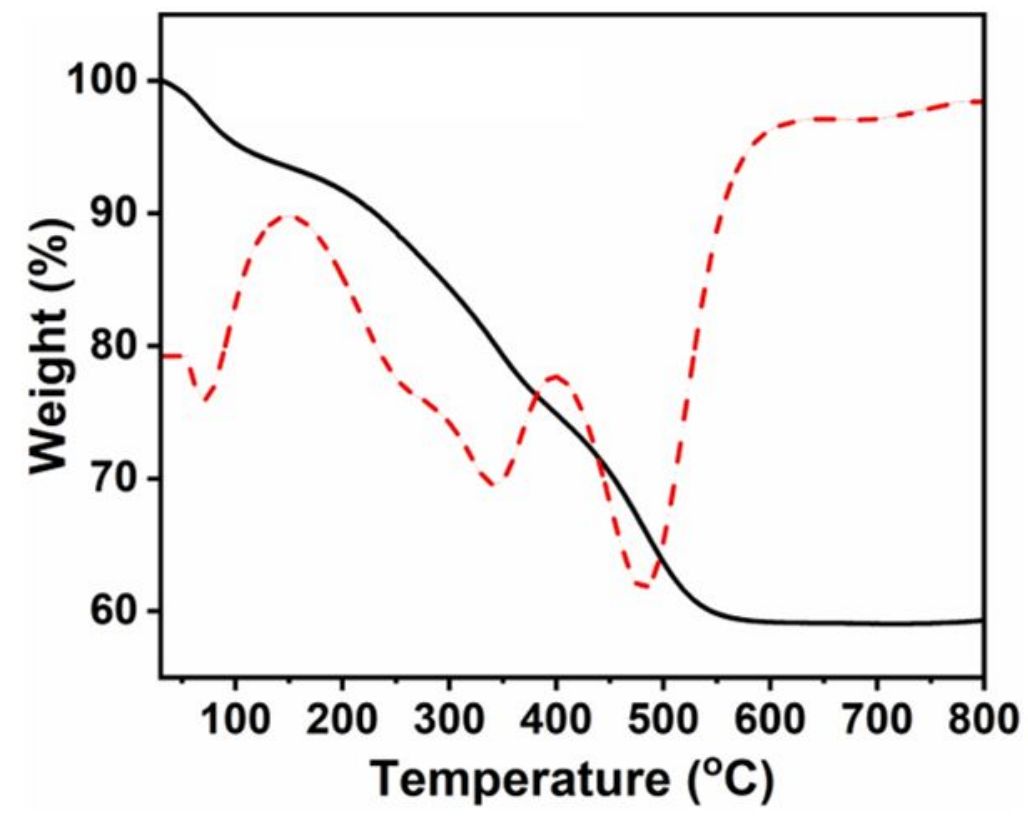

Figure S1 TG-DTA of PS@PMA- $\mathrm{ZrO}_{2}-\mathrm{PW}_{12}$.

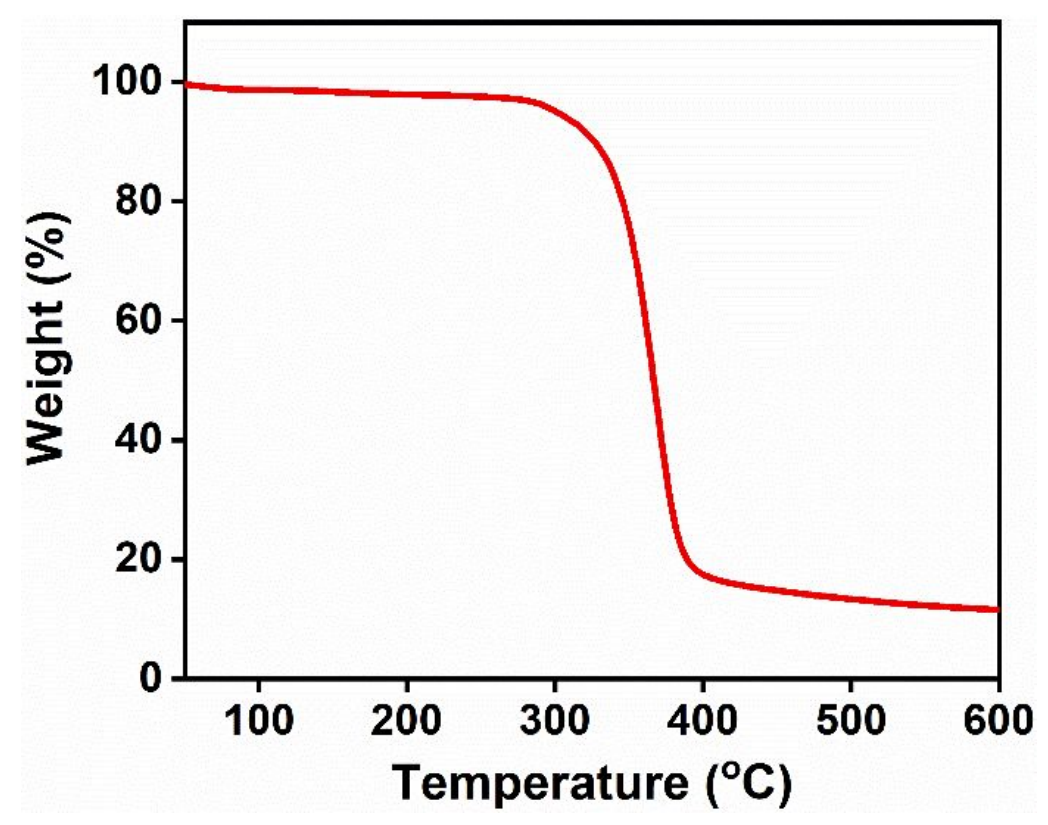

Figure S2 TG of CAP film. 


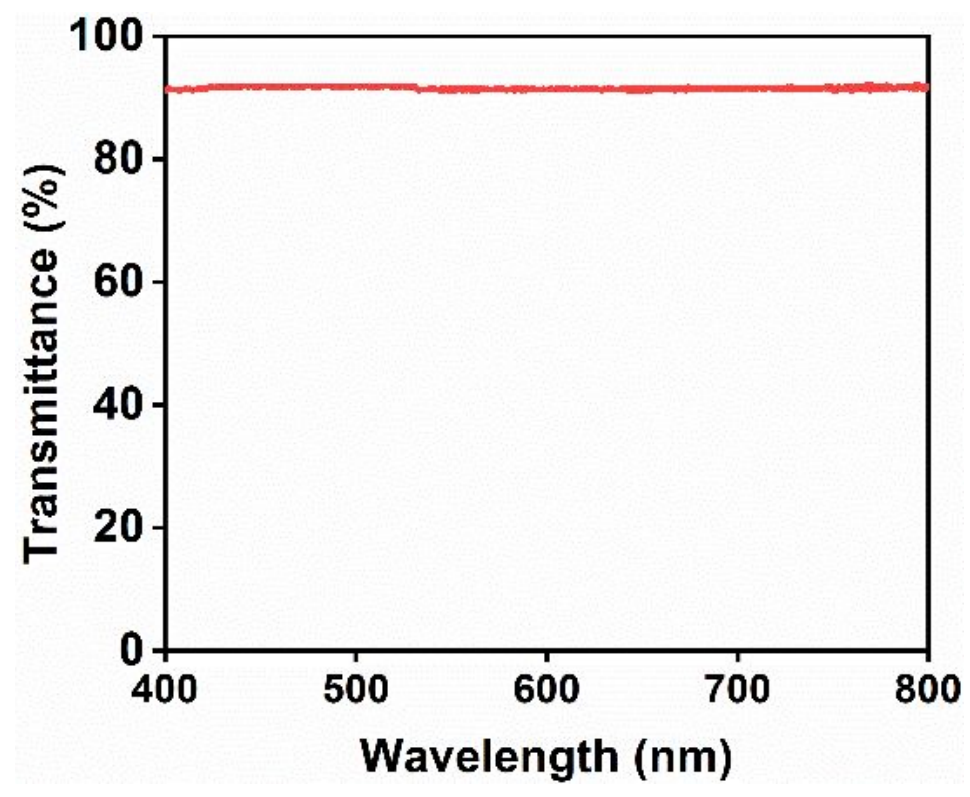

Figure S3 UV-vis spectrum of CAP film.

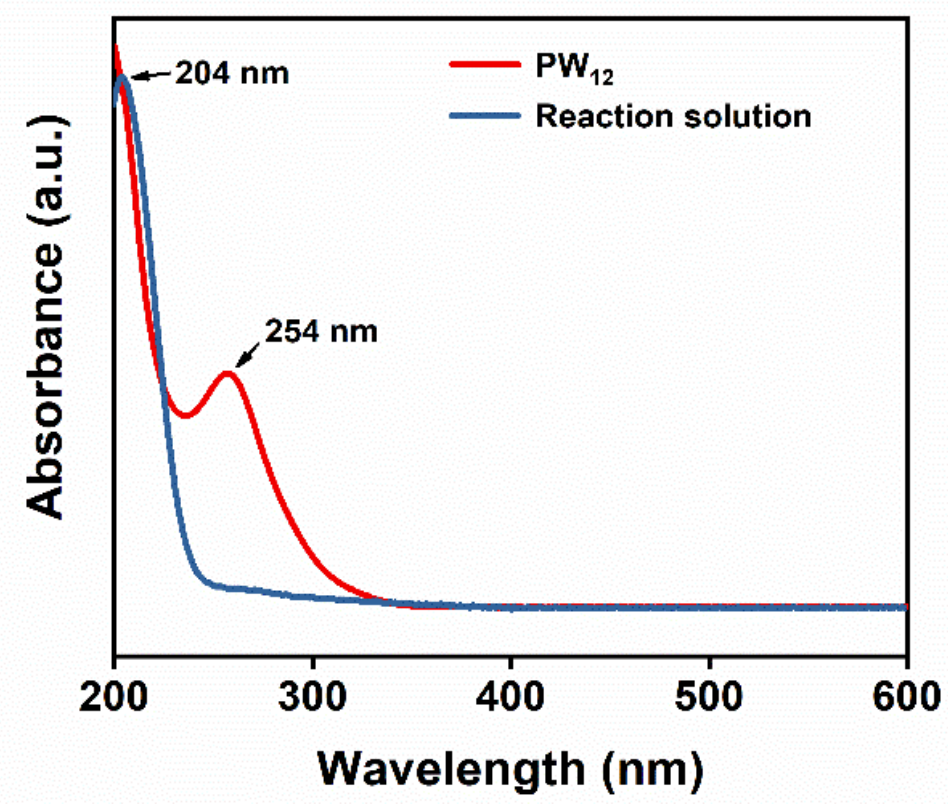

Figure S4 UV-vis spectra of $\mathrm{PW}_{12}$ and the reaction solution after separation of the usedPS@PMA-Z $\mathrm{rO}_{2}-\mathrm{PW}_{12}$. 
Table S1 Effect of HPAs on esterification activity, $M w$ and viscosity of CAP

\begin{tabular}{|c|c|c|c|c|c|c|c|}
\hline Catalyst & $D S_{\mathrm{A}}$ & $D S_{\mathrm{P}}$ & $D S_{\text {total }}$ & $\begin{array}{c}\mathrm{A} \\
(\%)\end{array}$ & $\begin{array}{c}\mathrm{P} \\
(\%)\end{array}$ & $M w$ & $\begin{array}{l}\text { Viscosity } \\
(\mathrm{mPa} \cdot \mathrm{s})\end{array}$ \\
\hline $\mathrm{PS} @ \mathrm{PMA}-\mathrm{ZrO}_{2}-\mathrm{PW}_{12}$ & 1.90 & 0.83 & 2.73 & 28.3 & 16.4 & 100644 & 320 \\
\hline $\mathrm{PS} @ \mathrm{PMA}-\mathrm{ZrO}_{2}-\mathrm{PMo}_{12}$ & 1.86 & 0.80 & 2.66 & 28.0 & 16.0 & 90020 & 210 \\
\hline PS@PMA-ZrO ${ }_{2}-\mathrm{SiW}_{12}$ & 1.50 & 0.90 & 2.40 & 23.4 & 18.6 & 29340 & 50 \\
\hline
\end{tabular}

Table S2 Effect of temperature on esterification activity, $M w$ and viscosity of CAP.

\begin{tabular}{|c|c|c|c|c|c|c|c|}
\hline $\begin{array}{l}\text { Temperature } \\
\left({ }^{\circ} \mathrm{C}\right)\end{array}$ & $D S_{\mathrm{A}}$ & $D S_{\mathrm{P}}$ & $D S_{\text {total }}$ & $\begin{array}{l}\text { A } \\
(\%)\end{array}$ & $\begin{array}{c}\mathrm{P} \\
(\%)\end{array}$ & $M w$ & $\begin{array}{l}\text { Viscosity } \\
(\mathrm{mPa} \cdot \mathrm{s})\end{array}$ \\
\hline 35 & 1.63 & 0.91 & 2.54 & 24.9 & 18.4 & 30425 & 50 \\
\hline 40 & 1.85 & 0.79 & 2.64 & 28.0 & 15.8 & 92882 & 230 \\
\hline 45 & 1.90 & 0.83 & 2.73 & 28.3 & 16.4 & 100644 & 320 \\
\hline 50 & 1.91 & 0.87 & 2.78 & 28.2 & 17.0 & 83640 & 190 \\
\hline 55 & 1.88 & 0.89 & 2.77 & 27.7 & 17.4 & 80015 & 180 \\
\hline
\end{tabular}


Table S3 Effect of esterification time on esterification activity, $M w$ and viscosity of CAP.

\begin{tabular}{cccccccc}
\hline $\begin{array}{c}\text { Time } \\
(\mathrm{h})\end{array}$ & $D S_{\mathrm{A}}$ & $D S_{\mathrm{P}}$ & $D S_{\text {total }}$ & $\begin{array}{c}\mathrm{A} \\
(\%)\end{array}$ & $\begin{array}{c}\mathrm{P} \\
(\%)\end{array}$ & $M w$ & $\begin{array}{c}\text { Viscosity } \\
(\mathrm{mPa} \cdot \mathrm{s})\end{array}$ \\
\hline 2.0 & 1.45 & 0.94 & 2.39 & 22.6 & 19.4 & 27510 & 40 \\
3.0 & 1.90 & 0.83 & 2.73 & 28.3 & 16.4 & 100644 & 320 \\
4.0 & 1.98 & 0.79 & 2.77 & 29.4 & 15.5 & 85220 & 200 \\
5.0 & 1.88 & 0.93 & 2.81 & 27.5 & 18.0 & 60040 & 140 \\
\hline
\end{tabular}

Table S4 Effect of anhydride/cellulose mass ratio on esterification activity, $M w$ and viscosity of CAP

\begin{tabular}{cccccccc}
\hline $\begin{array}{c}\text { Anhydride/cellulose } \\
\text { mass ratio }\end{array}$ & $D S_{\mathrm{A}}$ & $D S_{\mathrm{P}}$ & $D S_{\text {total }}$ & $\begin{array}{c}\mathrm{A} \\
(\%)\end{array}$ & $\begin{array}{c}\mathrm{P} \\
(\%)\end{array}$ & $\begin{array}{c}M w \\
(\mathrm{mPa} \cdot \mathrm{s})\end{array}$ \\
\hline $1: 1$ & 1.81 & 0.65 & 2.46 & 28.3 & 13.5 & 36300 & 70 \\
$3: 1$ & 1.83 & 0.81 & 2.64 & 27.6 & 16.2 & 70811 & 160 \\
$5: 1$ & 1.90 & 0.83 & 2.73 & 28.3 & 16.4 & 100644 & 320 \\
$8: 1$ & 1.85 & 0.84 & 2.69 & 28.1 & 16.9 & 87352 & 200 \\
$10: 1$ & 1.79 & 0.76 & 2.55 & 27.7 & 15.5 & 32074 & 60 \\
\hline
\end{tabular}

